\title{
Cola und Co.
}

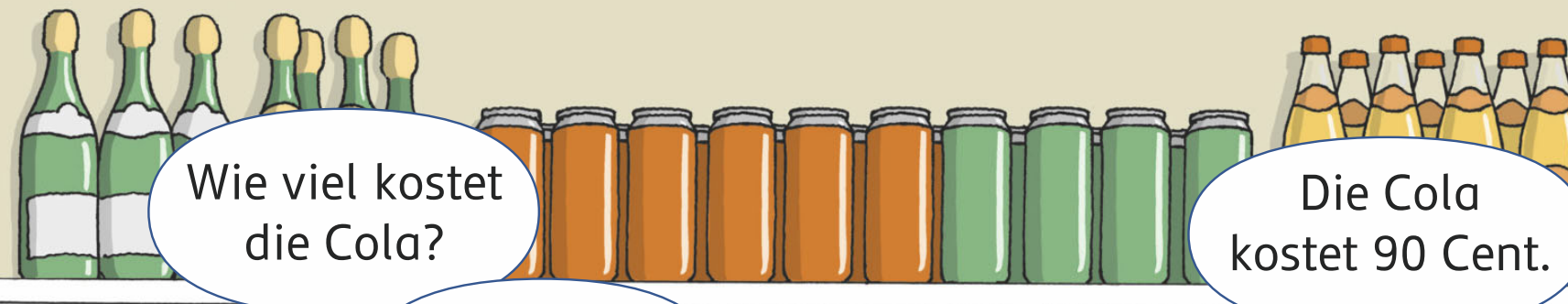

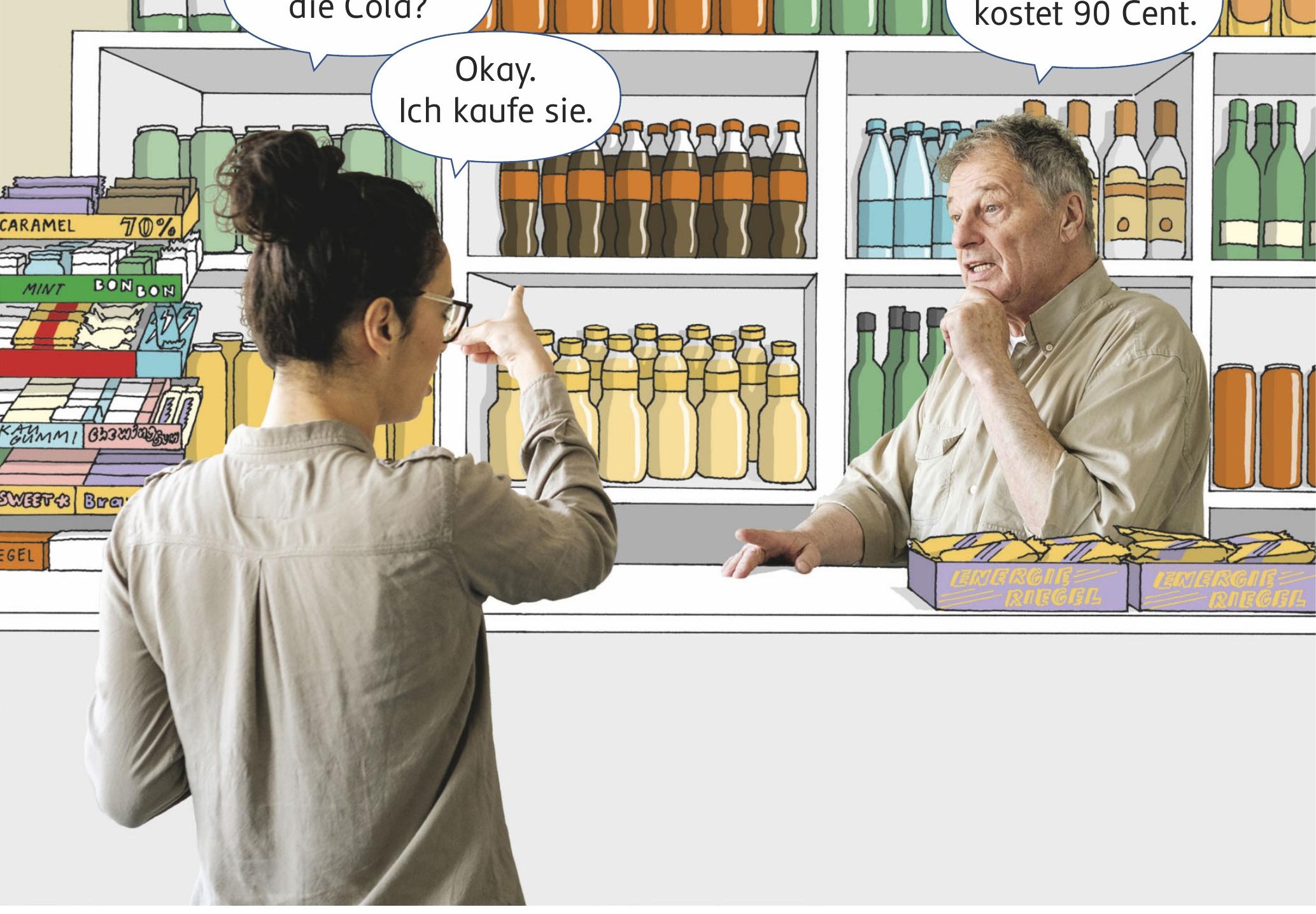

1 Was siehst du? Sprich.

2 Höre und sprich nach. (3) (1) 19

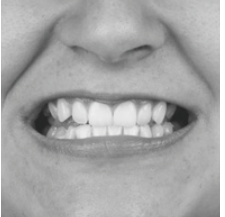

\section{Cola}




\section{Cola und Co.}

3a Sprich nach und fahre mit dem Finger nach. (2) (1)
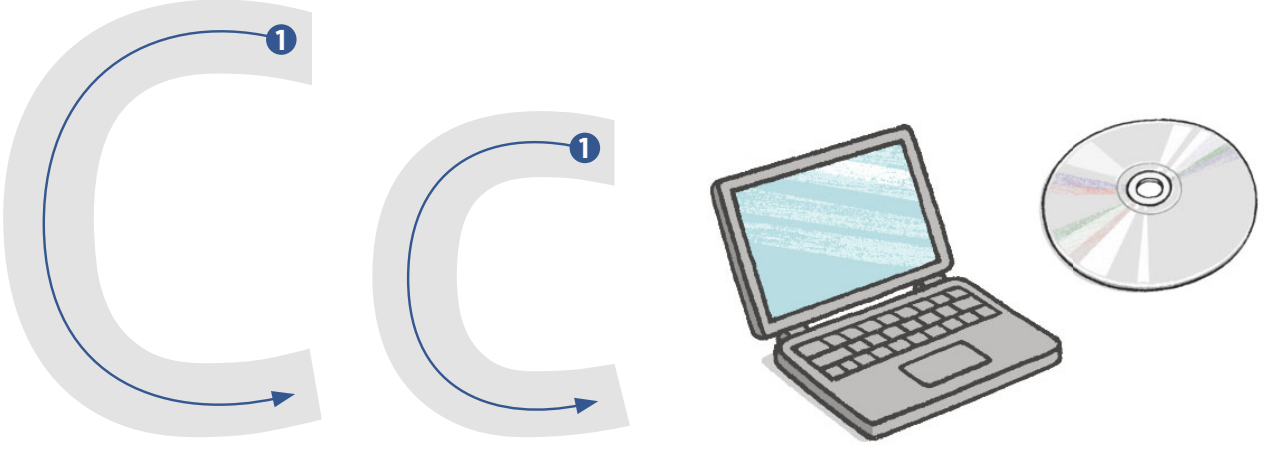

Vor a und o

spricht man c wie k.

Vor allen anderen Lauten spricht man c wie ts.

3b Schreibe.
C
C C
der Computer
die CD

C

3c Schreibe C c oder das ganze Wort nach.

$\begin{array}{llll}\text { die Cola } & \text { das Café } & \text { der Clown } & \text { die CD } \\ \text { der Comic } & \text { der Computer } & \text { die Creme } & \text { cool }\end{array}$

4 Zeichne die Silbenbögen ein und lies.

$\begin{array}{llll}\text { reparota } & \text { harumino } & \text { heurekal } & \text { dokumensa } \\ \text { milomegan } & \text { wimdurinter } & \text { columesti } & \text { funkulis }\end{array}$


5a Wie viel kostet das? Schreibe.
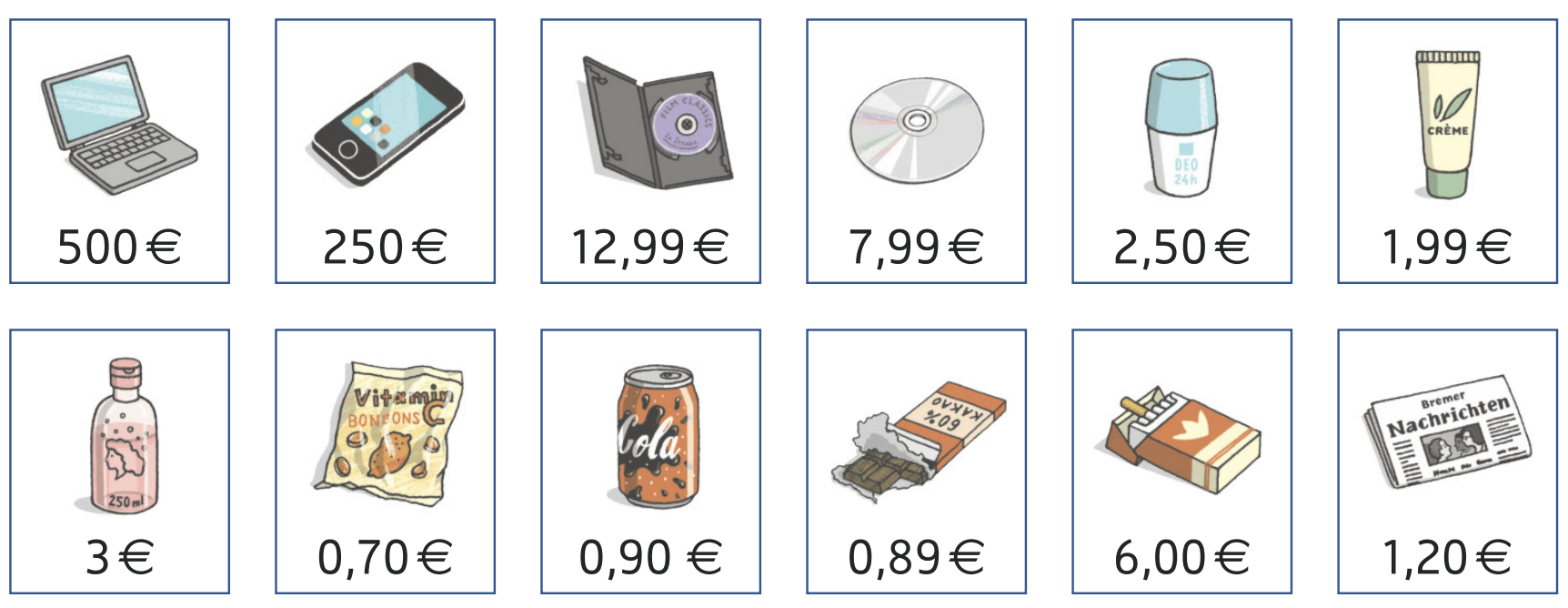

Wie viel kostet das Handy?

Wie viel kostet das Deo?

Wie viel kostet die DVD?

Wie viel kostet die Creme?

5b Was ist richtig? Höre und kreuze an.

(9) (t) 21
(1) $\square 250$ Euro $\square 350$ Euro
$2 \square 1$ Euro
$\square$ 1,50 Euro
$3 \square$ 0,99 Euro $\square$ 1,20 Euro
(4) $\square$ 12,99 Euro $\square$ 10,99 Euro 


\section{Cola und Co.}

6a Höre und sprich nach. (2) ()$_{22}$

- Wo finde ich Computer? • Computer finden Sie im Technikgeschäft.

- In welchem Stock? • Im zweiten Stock.

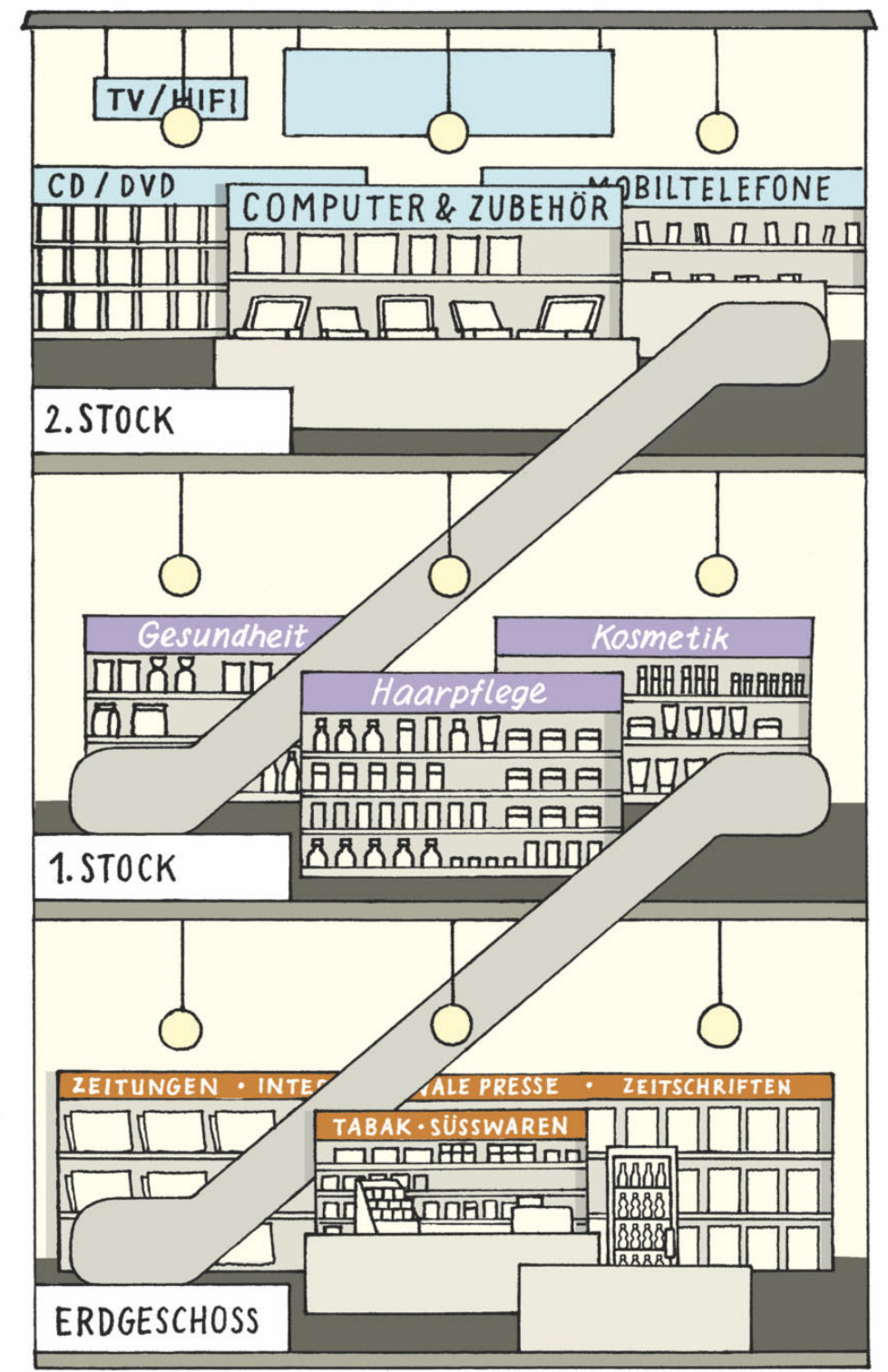

6b Sprecht zusammen.

09

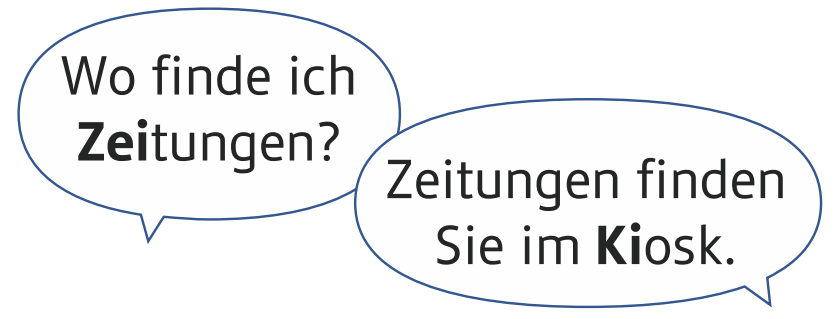

In welchem Stock?

Im Erdgeschoss. 
7a Was kauft Carla? Lies und schreibe. 을

\begin{tabular}{l}
\hline Hallo. Ich bin Carla. \\
Ich bin 17 Jahre alt und gehe in die \\
Heute kaufe ich viele Dinge. \\
Ich kaufe einen \\
Und ich kaufe ein \\
Deine Carla
\end{tabular}

7b Schreibe die Antwort.

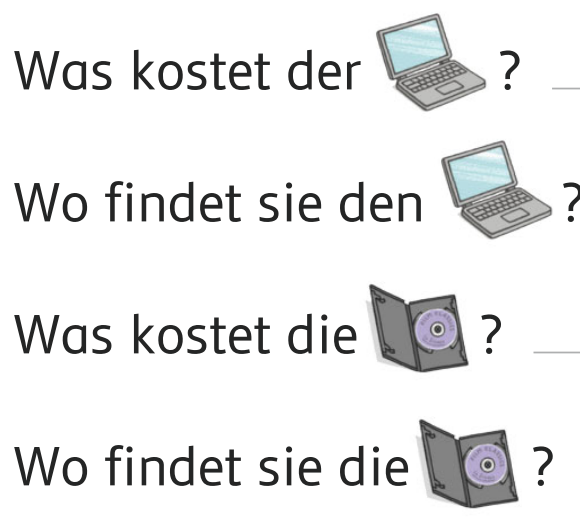

7c Was kaufst du? Schreibe eine E-Mail.

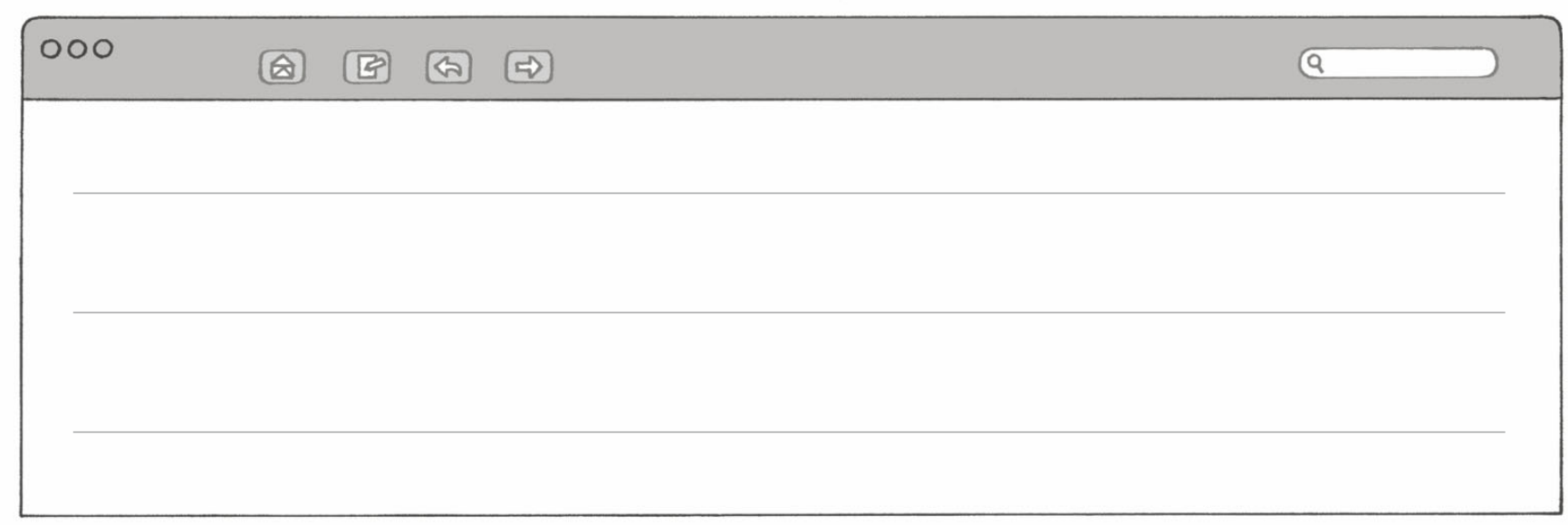




\section{Cola und Co.}

8a Wo findest du das im Kaufhaus? Schreibe.

$\begin{array}{llll}\text { die Creme } & \text { der Schal } & \text { der Zucker } & \text { der Computer } \\ \text { der Salat } & \text { das Parfum } & \text { das Handy } & \text { die Jeans }\end{array}$

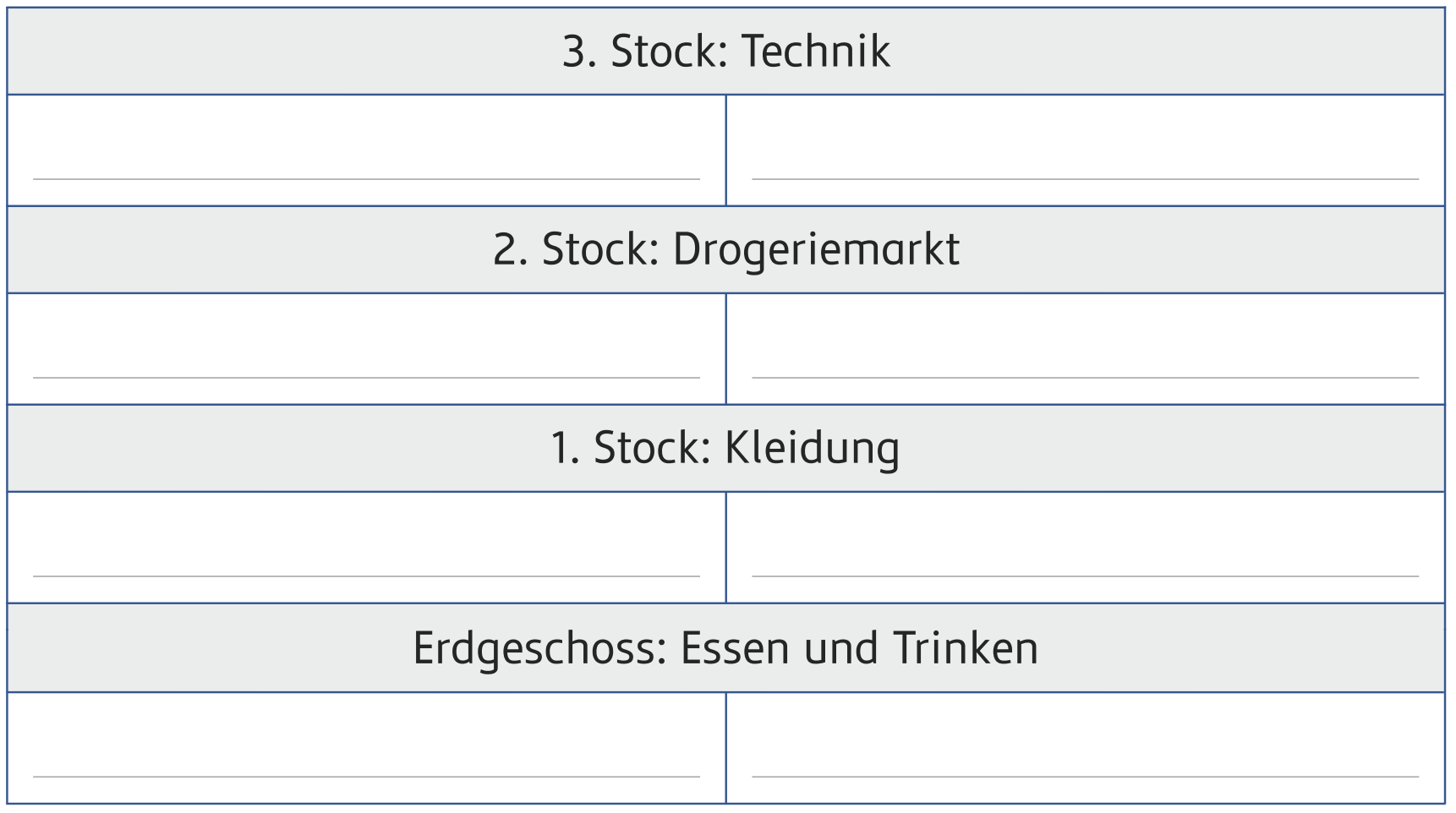

8b Du hast 100 Euro. Welche fünf Dinge kaufst du? Schreibe Sätze. 


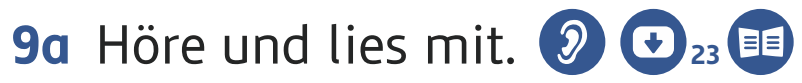

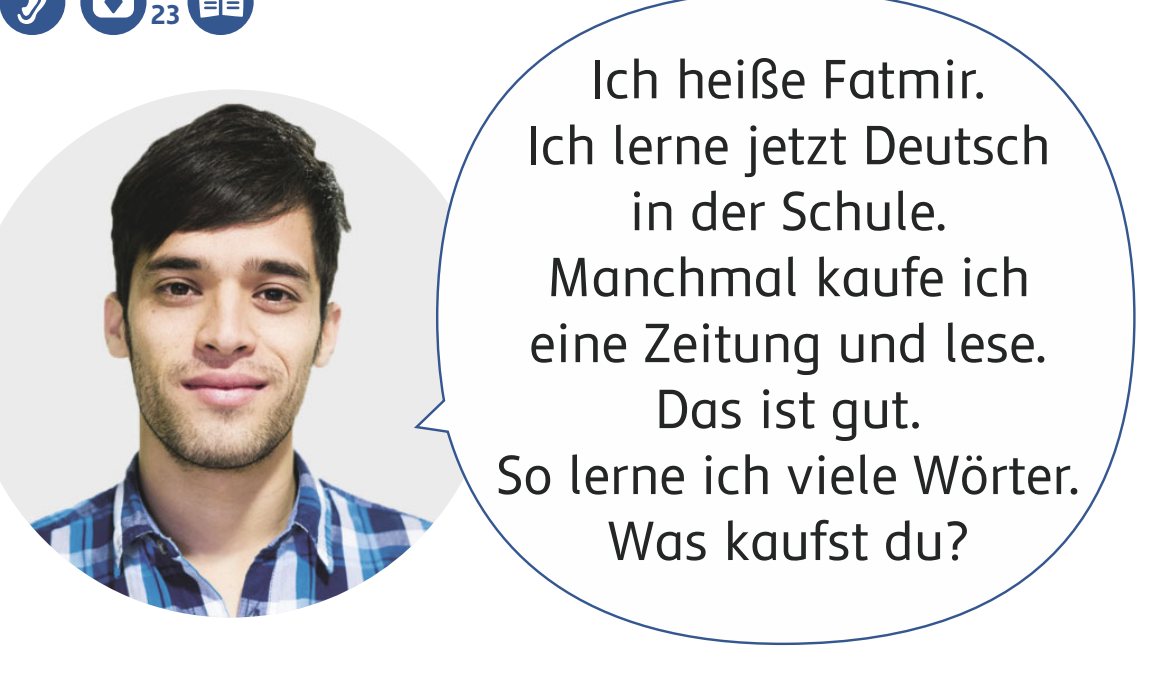

9b Meine Aufgabe für dich. Welches Wort ist falsch?

Wie Was kaufst du? Ich kaufe eine viele Zeitung.

Wo finde ich du Zeitungen? Du findest die

Zeitungen im Kiosk finden. Wie viel kostet die

Zeitung? Die das Zeitung Kostet 1 Euro.

9c Schreibe eine eigene Aufgabe. 
$\star$ Verbinde.

die Zahnbürste

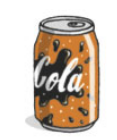

das Deo

50 Cent

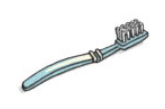

der Kiosk

die Cola

$\star \star$ C oder K? Schreibe.

_omic

ola iosk

affee

\section{das Shampoo \\ die CD \\ der Computer}

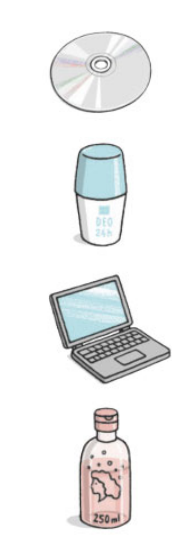

\section{8}

$\begin{array}{cr}\text { _iosk } & \text { reme } \\ \text { affee } & \text { _omputer }\end{array}$

$\star \star \star$ Schreibe.

- Hal _ o. Ich möchte einen Co___ uter kaufen.

- Guten T__ _ Ja bitt__. Wir hab__ viele Computer.

- Wo finde i__ die Computer?

- D___ Computer finden Sie im dritten Stock.

- Sehr gut. Und wie vi __ _ kosten d___ Computer?

- Circa 500 Euro. Fra _ en Sie bitte im drit____ Stock.

- Okay. Dan__ _ schön.

- Bitte sch_n. 
Finde die Wörter und schreibe. Welche Wörter schreibt man groß?

holacolameisumcdkschacaférukccoolbrrcomputerxbiulmcremeschäusde okonepreishuidnumsulzahnbürsteoompashgerdgeschossgutzdhumsuli

Cola

$\star \star$ Sortiere nach dem Alphabet und schreibe.
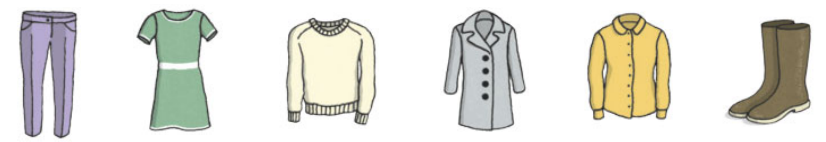

$\star \star \star$ Lies und antworte. 目

Ich bin Carla und komme aus Costa Rica. Ich bin 17 Jahre alt und gehe in die Schule. Heute kaufe ich viele Dinge. Ich kaufe einen Computer und eine DVD. Und ich kaufe ein Deo und eine Cola.

Wie heißt das Mädchen?

Woher kommt sie?

Was kauft sie? 


\section{Mein Wortschatz}

Schreibe.

die $C D$, die $C D s$

die Creme, die Cremes

das Deo, die Deos

die DVD, die DVDs

das Shampoo, die Shampoos

die Zahnbürste, die Zahnbürsten

der Stock

das Erdgeschoss (EG)

der Preis, die Preise

das Technikgeschäft, die Technikgeschäfte

der Drogeriemarkt, die Drogeriemärkte

Lies und sprich.

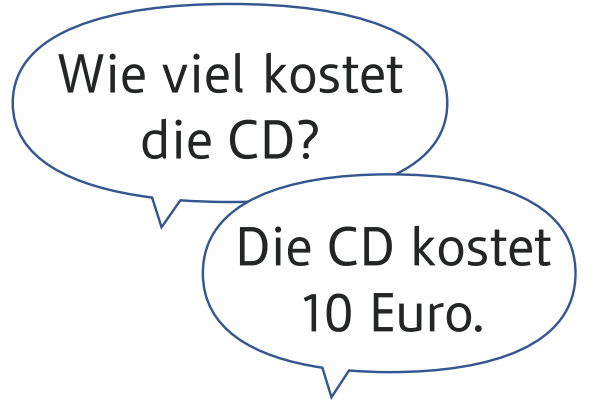

Wo finde ich

die CD?

Du findest die CD im zweiten Stock. 
Ordne Wort und Bild.
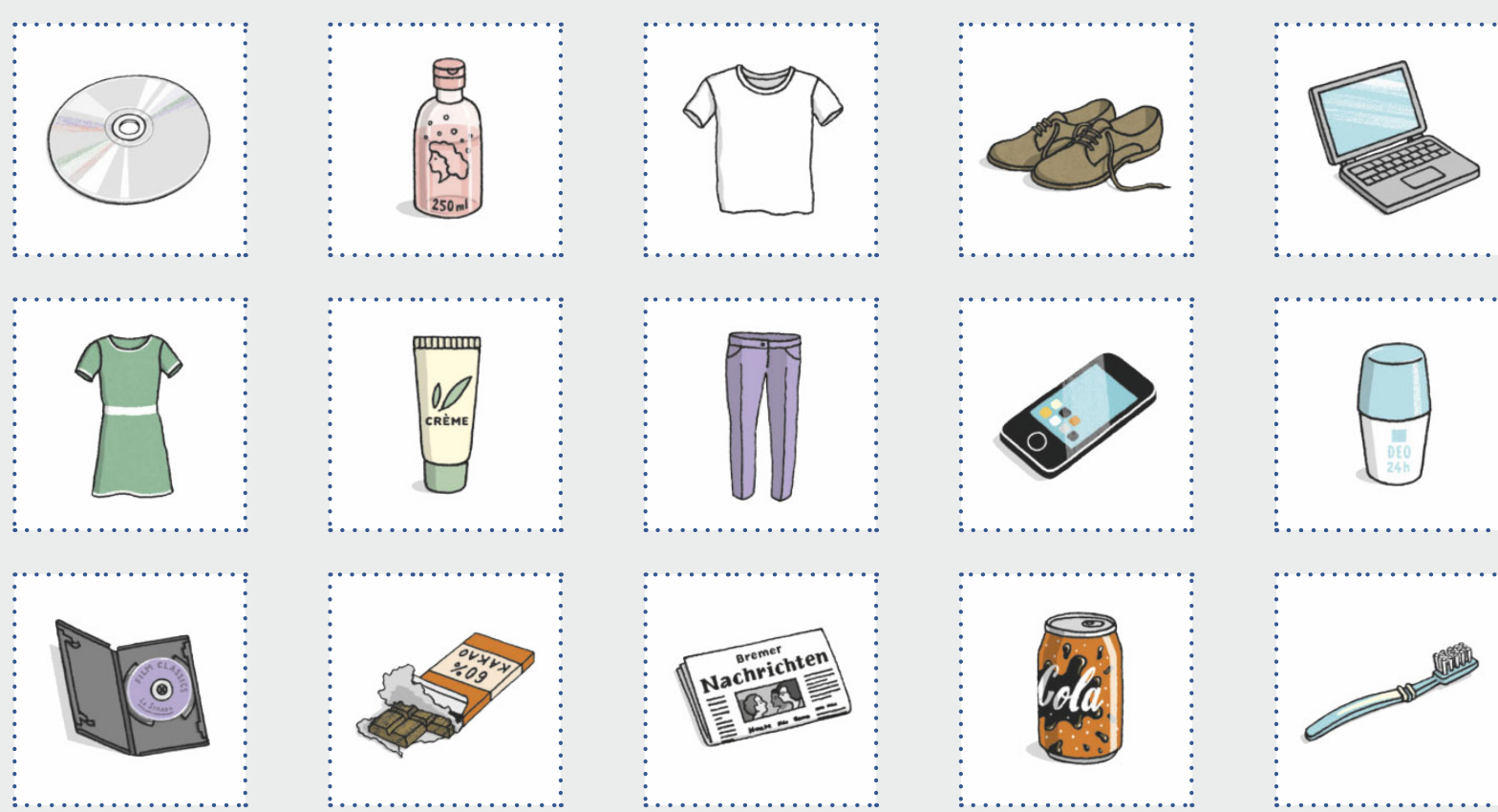

\begin{tabular}{|c|c|c|}
\hline die CD & das Shampoo & das T-Shirt \\
\hline die Schuhe & der Computer & das Kleid \\
\hline die Hose & die Creme & das Handy \\
\hline die Zahnbürste & das Deo & die Cola \\
\hline die DVD & die Schokolade & die Zeitung \\
\hline
\end{tabular}


Open Access Dieses Kapitel wird unter der Creative Commons Namensnennung - Nicht kommerziell - Keine Bearbeitung 4.0 International Lizenz (http://creativecommons.org/licenses/by-nc-nd/4.0/deed.de) veröffentlicht, welche die nicht-kommerzielle Nutzung, Vervielfältigung, Verbreitung und Wiedergabe in jeglichem Medium und Format erlaubt, sofern Sie den/die ursprünglichen Autor(en) und die Quelle ordnungsgemäß nennen, einen Link zur Creative Commons Lizenz beifügen und angeben, ob Änderungen vorgenommen wurden. Die Lizenz gibt Ihnen nicht das Recht, bearbeitete oder sonst wie umgestaltete Fassungen dieses Werkes zu verbreiten oder öffentlich wiederzugeben.

Die in diesem Kapitel enthaltenen Bilder und sonstiges Drittmaterial unterliegen ebenfalls der genannten Creative Commons Lizenz, sofern sich aus der Abbildungslegende nichts anderes ergibt. Sofern das betreffende Material nicht unter der genannten Creative Commons Lizenz steht und die betreffende Handlung nicht nach gesetzlichen Vorschriften erlaubt ist, ist auch für die oben aufgeführten nicht-kommerziellen Weiterverwendungen des Materials die Einwilligung des jeweiligen Rechteinhabers einzuholen. 